\title{
MICA enhances sensitivity to cisplatin in patients with extensive small cell lung cancer via downregulation of ABCG2
}

\author{
YUFENG WU $^{1 *}$, HONG TANG $^{1 *}$, RUIRUI SI $^{2}$, SUHUA XIA $^{3}$, RUILIN WANG $^{1}$ and QIMING WANG ${ }^{1}$ \\ ${ }^{1}$ Department of Internal Medicine, Henan Cancer Hospital, The Affiliated Cancer Hospital of Zhengzhou University, \\ Zhengzhou, Henan 450008; ${ }^{2}$ Department of Health Center, Henan Airport Group Co., Ltd., Zhengzhou, Henan 450000; \\ ${ }^{3}$ Department of Oncology, The First Affiliated Hospital of Soochow University, Suzhou, Jiangsu 215006, P.R. China
}

Received November 8, 2019; Accepted April 9, 2020

DOI: $10.3892 / \mathrm{ol} .2020 .11646$

\begin{abstract}
Immunotherapy utilizing natural killer cell-activated receptor natural-killer group-2 member D ligands (NKG2DLs) has had preclinical success in the treatment of small cell lung cancer. The present study aimed to investigate the association between NKG2Ls and chemoresistance. The mRNA expression of six NKG2DLs associated with progression-free survival time (PFS) and first-line chemotherapy were assessed in the present study. Major histocompatibility complex class I polypeptide-related sequence A (MICA)-overexpressing NCI-H446 cell line was constructed, and the mRNA expression levels of 11 genes associated with chemotherapy sensitivity were determined. The results demonstrated that MICA was positively and significantly associated with PFS. Furthermore, MICA expression was 1.6 times higher in
\end{abstract}

Correspondence to: Professor Yufeng $\mathrm{Wu}$ or Professor Qiming Wang, Department of Internal Medicine, Henan Cancer Hospital, The Affiliated Cancer Hospital of Zhengzhou University, 127 Dongming Road, Zhengzhou, Henan 450008, P.R. China

E-mail: yufengwu@vip.qq.com

E-mail: qimingwang1006@163.com

*Contributed equally

Abbreviations: NKG2D, natural killer cell-activated receptor natural-killer group-2 member D; NKG2DLs, NKG2D ligands; ABCG2, ATP binding cassette subfamily G member 2; PFS, progression-free survival; SCLC, small cell lung cancer; QCR, quick chemoresistance; DCR, delayed chemotherapy resistance; MHC, major histocompatibility complex; MIC, MHC class I chain-related protein; ULBP, UL16-binding protein; DPYD, dihydropyrimidine dehydrogenase; GSTT1, glutathione S-transferase 01 ; GSTp1, glutathione S-transferase pi 1; GSTM1, glutathione S-transferase mu 1; ADH1A, alcohol dehydrogenase 1A (class I), alpha polypeptide; PPARA, peroxisome proliferator activated receptor alpha

Key words: major histocompatibility complex class I polypeptiderelated sequence $\mathrm{A}$, extensive small cell lung cancer, natural killer cell-activated receptor natural-killer group-2 member D ligands, ATP binding cassette subfamily $G$ member 2 , progression-free survival patients with prolonged PFS compared with the rapid chemoresistance group. ATP binding cassette subfamily $\mathrm{G}$ member 2 (ABCG2) mRNA expression was associated with chemotherapy resistance and significantly downregulated in the cell line overexpressing MICA. Moreover, following addition of nicardipine (an ABCG2 inhibitor), chemotherapeutic sensitivity increased in the MICA-overexpressing cell line. Taken together, the results of the present study suggested that MICA may enhance the chemosensitivity of patients with extensive small cell lung cancer by downregulating ABCG2.

\section{Introduction}

Lung cancer is a malignant tumor with extremely high incidence (36.71 per 100,000) and mortality rates $(28.49$ per $100,000)$ in China in 2018 (1). Small cell lung cancer (SCLC) is a common subtype of lung cancer. Currently, 4-6 cycles of platinum-based chemotherapy (cisplatin or carboplatin) remains the primary treatment option for patients with extensive SCLC (2). Despite recent advancements in novel chemotherapy regimens and improvements in the use of immunotherapy, $>75 \%$ of patients with SCLC relapse within 6 months (3), and the overall 5-year survival rate of patients with SCLC remains $<15 \%(4,5)$. Immunotherapy has gained recent interest as a validated clinical treatment for several types of cancer, including SCLC (6). However, given that immunotherapy is not a first-line treatment for SCLC, the majority of patients who receive immunotherapy have previously undergone chemotherapy several times (7). Thus, understanding the association between chemotherapy and immunity is of great importance.

Natural killer (NK) cells serve a critical role in the tumor cell immune response and their function is regulated via signals initiated by a variety of activating and inhibitory receptors on NK cells (8). NK cell-activated receptor natural-killer group-2 member D (NKG2D) is expressed on all NK cells (9). NKG2D ligands (NKG2DLs), as homologues of major histocompatibility complex (MHC) I molecules in human tissues, include two families: MHC I chain-related proteins (MICs) $(10,11)$ and UL16-binding proteins (ULBPs) $(12,13)$. MHC class I polypeptide-related sequence A (MICA) is predominantly expressed in epithelial-derived tumor cells, including lung, breast, renal and ovarian cancer cells, as well as in certain melanoma cells (14). 
The combination of NKG2D with MICA on the tumor surface can directly activate NK cells and enhance the killing activity of NK cells against tumor cells (15). Simultaneously, NKG2D, which recognizes non-MHC class I molecules, serves a crucial role in mediating the immune response of NK cells when recognizing and attacking tumor cells (16). Downregulation of NKG2D decreases the sensitivity of NK cells to lung cancer cells, following MHC-I deletion. Furthermore, a previous study have that the expression of these ligands are associated with enhanced antibody-dependent cell-mediated cytotoxic activity, which is one of the fundamental molecular mechanisms underlying the antitumor effect of antibody therapeutics (17). This topic is of considerable interest as the potential to manipulate NKG2DL expression may offer promise in the future treatment of different types of tumor.

To the best of our knowledge, limited research has focused on the association between chemotherapy and ligands. Thus, the present study aimed to investigate the expression of NKG2DLs in tissue samples of patients with SCLC to determine the association between NKG2DLs and chemoresistance.

\section{Materials and methods}

Clinical characteristics and data collection of patients. The present study was approved by the Ethics Committee of Zhengzhou University (approval no. 2014079), and performed in accordance with The Declaration of Helsinki. Written informed consent was provided by all patients prior to study enrolment. Biopsy tissue samples were collected from 115 patients with advanced SCLC who received platinum-based chemotherapy at The Affiliated Cancer Hospital of Zhengzhou University (Zhengzhou, China), between January 2014 and March 2018. The median age of the patients with advanced SCLC who were included in the study was 65.32 years (range, 56.25-77.14 years), of which 78 (67.83\%) were male and 37 $(32.17 \%)$ were female. Histological diagnosis was based on H\&E and immunohistochemical staining, according to the 2015 World Health Organization criteria (18). The inclusion criteria were as follows: i) Patients clinically diagnosed with SCLC; ii) patients $\geq 18$ years old; iii) patients diagnosed by biopsy; and iv) patients pathologically diagnosed with advanced SCLC. Patients who had a history of other malignant tumors, or who had previously received radiotherapy or chemotherapy were excluded from the present study. The clinical characteristics of patients with SCLC are presented in Table I.

Imaging examination (chest-abdomen CT and head MRI) was performed regularly every two chemotherapy cycles until tumor progression was observed. Routine blood examination, assessment of liver-kidney function and ECG prior to chemotherapy were performed for each patient. Chemotherapy response was evaluated according to the Response Evaluation Criteria in Solid Tumors (version 1.1) (19). Progression-free survival (PFS) was measured following first-line chemotherapy. PFS was defined from the beginning of chemotherapy until disease progression or mortality from any cause. The method of follow-up was via regular outpatient assessment every 3 weeks. The follow-up period was between 1st February 2014 and 31st March 2018.

Reverse transcription-quantitative (RT-q)PCR. Fresh lung puncture tissue samples were immediately immersed in
RNAlater Stabilization reagent (Qiagen AB) and stored at $-20^{\circ} \mathrm{C}$ until RNA extraction. Total RNA was extracted using TRIzol $^{\circledR}$ reagent (Invitrogen; Thermo Fisher Scientific, Inc.), according to the manufacturer's protocol. Total RNA (1 $\mu \mathrm{g})$ was reverse transcribed into cDNA using a OneStep RT-PCR kit, according to the manufacturer's protocol (Tiangen Biotech Co., Ltd.). Temperature protocol were carried out at $25^{\circ} \mathrm{C}$ for $5 \mathrm{~min}$ and $42^{\circ} \mathrm{C}$ for $30 \mathrm{~min}$ and 85 for $5 \mathrm{~min}$. qPCR was subsequently performed to determine the mRNA expression of the six selected NKG2DLs [MICA, MHC class I polypeptide-related sequence B (MICB), UL16-binding proteins 1 (ULBP1), UL16-binding proteins 2 (ULBP2), UL16-binding proteins 3 (ULBP3), UL16-binding proteins 4 (ULBP4)] (14) or 11 selected genes according to previous research [ATP binding cassette subfamily B member 1 (ABCB1), ATP binding cassette subfamily $\mathrm{C}$ member 1 (ABCC1), ATP binding cassette subfamily $\mathrm{C}$ member 2 (ABCC2), ATP binding cassette subfamily G member 2 (ABCC2), ATP binding cassette subfamily $\mathrm{C}$ member 9 (ABCC9), dihydropyrimidine dehydrogenase (DPYD), glutathione S-transferase $\theta 1$ (GSTT1), glutathione S-transferase $\pi 1$ (GSTp1), glutathione S-transferase mu 1 (GSTM1), alcohol dehydrogenase 1A class I $\alpha$ polypeptide (ADH1A), peroxisome proliferator activated receptor $\alpha$, (PPARA)] $(20,21)$, using FastStart Universal SYBR Green Master mix (Roche Diagnostics GmbH) on a StepOne Plus real-time PCR system (Applied Biosystems; Thermo Fisher Scientific, Inc.). 115 patients' whole blood samples were used as the control. The primer sequences used for qPCR are listed in Table S1, and the final concentration of each primer in a reaction was $0.5 \mu \mathrm{M}$. The following thermocycling conditions were used for qPCR: Initial denaturation at $95^{\circ} \mathrm{C}$ for $3 \mathrm{~min} ; 35$ cycles of amplification (denaturation at $95^{\circ} \mathrm{C}$ for $30 \mathrm{sec}$, annealing at $60^{\circ} \mathrm{C}$ for $30 \mathrm{sec}$, elongation at $72^{\circ} \mathrm{C}$ for $20 \mathrm{sec}$ ) and a final elongation at $68^{\circ} \mathrm{C}$ for $10 \mathrm{~min}$. Data were analyzed with the $2^{-\Delta \Delta \mathrm{Cq}}$ method (22).

Cell culture. The NCI-H446 cell line, which was kindly provided by Dr ZeLai He from Medical Oncology of The First Affiliated Hospital of Bengbu Medical College (Bengbu, China), was cultured in RPMI-1640 complete medium (HyClone; GE Healthcare Life Sciences) supplemented with $10 \%$ FBS, $2 \mathrm{mM}$ glutamine, $100 \mu \mathrm{g} / \mathrm{ml}$ streptomycin and $100 \mathrm{U} / \mathrm{ml}$ penicillin (Invitrogen; Thermo Fisher Scientific, Inc.) at $37^{\circ} \mathrm{C}$ and $5 \% \mathrm{CO}_{2}$.

Overexpression of MICA. The full-length MICA gene (Shanghai GeneChem Co., Ltd.) was subcloned into the pCDH-CMV-MCS lentivector (System Biosciences, https://systembio.com/shop/pcdh-cmv-mcs-cdna-single-prom oter-cloning-and-expression-lentivector/), between the NheI and SalI sites. The infectious lentiviral particles were generated and concentrated as previously described (21). A total of $2 \times 10^{5} \mathrm{NCI}-\mathrm{H} 446$ cells were transduced with the recombinant lentivirus carrying the human MICA gene or an empty vector to generate the stable MICA-overexpressing cells (OE-MICA) or control cells (VC-MICA), respectively.

MTT assay. To assess the chemosensitivity of tumor cells, cell viability was measured via an MTT assay (Promega Corporation). Cell suspensions were cultured in RPMI-1640 
Table I. Clinical characteristics of patients with small cell lung cancer $(n=115)$.

\begin{tabular}{lccc}
\hline Characteristic & Patient, $\mathrm{n}$ & Cases, $\%$ & mPFS time, months \pm SD \\
\hline Total, $\mathrm{n}$ & 115 & & $6.29 \pm 2.24$ \\
Sex & & & \\
Male & 78 & 67.83 & $6.02 \pm 1.78$ \\
Female & 37 & 32.17 & $6.75 \pm 1.90$ \\
Age, years & & & \\
$<57$ & 77 & 66.96 & $6.34 \pm 2.03$ \\
$\geq 57$ & 38 & 33.04 & $5.97 \pm 1.35$ \\
Smoker & & & \\
Never & 46 & 40.00 & $6.38 \pm 2.27$ \\
Previous/Current & 69 & 60.00 & $5.82 \pm 1.75$ \\
Metastasis site & & & \\
Brain & 21 & 18.26 & 5.063 \\
Liver & 18 & 15.65 & $4.44 \pm 1.93$ \\
Distant lymphoid & 35 & 30.43 & $6.60 \pm 2.11$ \\
Others & 41 & 35.65 & $7.20 \pm 1.96$ \\
\hline
\end{tabular}

${ }^{\mathrm{a}} \mathrm{P}<0.05$ (liver vs. brain), ${ }^{\mathrm{b}} \mathrm{P}<0.001$ vs. others. ${ }^{\mathrm{c}}$ Others include bone, pleura, adrenal gland, etc. mPFS, median progression-free survival.

medium(ThermoFisherScientific,Inc.)in96-well flat-bottomed microtiter plates at a density of $1 \times 10^{4}$ cells/well and incubated overnight at $37^{\circ} \mathrm{C}$. For the nicardipine combination experiment, the concentration of nicardipine (Sigma-Aldrich; Merck $\mathrm{KGaG}$ ) in the culture medium was adjusted to $10 \mu \mathrm{M}$ and incubated at $37^{\circ} \mathrm{C}$ for $15 \mathrm{~min}$ in advance.

The following concentrations of cisplatin (Sigma-Aldrich; Merck $\mathrm{KGaG}$ ) were used: $10^{-7} \mathrm{M}, 10^{-6} \mathrm{M}, 10^{-5} \mathrm{M}, 10^{-4} \mathrm{M}$ and $10^{-3} \mathrm{M}$. All experiments were performed in triplicate, for each concentration. NCI-H446 cells cultured in RPMI-1640 medium were used as the blank controls. MTT solution was added $\left(1 \mathrm{mg} / \mathrm{ml}\right.$ per well) and incubated at $37^{\circ} \mathrm{C}$ for $48 \mathrm{~h}$. Cell viability was subsequently analyzed at a wavelength of $590 \mathrm{~nm}$, using a spectrophotometric microplate reader (Bio-Rad Laboratories, Inc.). The response percentage of cell viability to different drug concentrations was calculated as the inhibition rate (average absorbance of treatment wells/control wells $\times 100 \%)$.

Western blotting. Cell or tumor lysates were obtained and equal amounts of protein lysates from each sample were diluted with loading buffer, denatured, and separated by $10 \%$ sodium dodecyl sulfate-polyacrylamide gel electrophoresis (SDS-PAGE) followed by protein transfer to polyvinylidene fluoride membranes (PVDF). After incubation in a blocking solution (5\% nonfat milk powder) in TBST buffer $(10 \mathrm{mM}$ Tris-HCl, pH 8.0, $150 \mathrm{mM} \mathrm{NaCl}$, and $0.1 \%$ Tween-20) for $1 \mathrm{~h}$ at room temperature, the membranes were immunoblotted overnight with primary monoclonal antibodies (Abs) against either ABCG2 (Santa Cruz Biotechnology Inc.) or Actin at a 1:1,000 dilution at $4^{\circ} \mathrm{C}$. The membranes were then incubated for $2 \mathrm{~h}$ at room temperature with the appropriate secondary antibody (1:1,000 dilution). The protein antibody complex was detected by an enhanced chemiluminescence detection system. The protein expression was quantified by ImageJ software (http:/rsbweb.nih.gov/ij/).

GeneMANIA prediction. GeneMANIA datasets (http://genemania.org) were downloaded from to show the interaction between 17 genes, including the six selected NKG2DLs (MICA, MICB, ULBP1, ULBP2, ULBP3, ULBP4) (14) or 11 selected genes according to previous research (ABCB1, ABCC1, ABCC2, ABCC2, ABCC9, DPYD, GSTT1, GSTp1, GSTM1, ADH1A, PPARA) $(20,21)$, including: Co-expression data from Gene Expression Omnibus (23); physical and genetic interaction data from Biological General Repository for Interaction Datasets (BioGRID) (24); predicted protein interaction data, based on orthology, from I2D (25) and pathway and molecular interaction data from Pathway Commons, which contains data from BioGRID (24), Memorial Sloan-Kettering Cancer Center, Human Protein Reference Database (26), HumanCyc (27), Systems Biology Center New York, IntAct (28), MINT (29), NCI-Nature Pathway Interaction Database (30) and Reactome $(31,32)$.

Statistical analysis. Statistical analysis was performed using SPSS software (version 19; IBM Corp.). Associations between NKG2DLs and PFS were assessed using the coefficient of determination $\left(\mathrm{R}^{2}\right)$. The half maximal inhibitory concentration $\left(\mathrm{IC}_{50}\right)$ values were calculated using a non-linear regression model within GraphPad Prism software (version 6.0; GraphPad Software Inc.). Pearson's correlation analysis was performed the correlation analysis of NKG2DLs and PFS. Unpaired Student's t-tests were used to compare differences between two groups, while one-way ANOVA followed by Bonferroni's correction was used to compare cell viability between multiple groups, at $10 \mu \mathrm{mol} / 1$ of carboplatin. $\mathrm{P}<0.05$ (two-tailed) was considered to indicate a statistically significant difference. 


\section{Results}

Clinical information of patients with extensive SCLC. The age range of the 115 patients with extensive SCLC was between 46.5 to 68.9 years and the total median PFS (mPFS) associated with first-line chemotherapy was $6.29 \pm 2.24$ months. There were 78 men $(67.83 \%)$ and 37 women $(32.17 \%)$ in the study group. There were no significant differences between the two groups according to the median age (57 years; 77 patients $<57$ and 38 patients $\geq 57$ ). There were 69 smokers $(60.00 \%)$. As presented in Table I, smoking and metastasis site were significantly associated with the mPFS in relation to chemotherapy.

The location of tumor metastasis was significantly associated with the efficacy of chemotherapy and the rates of metastasis to the brain, liver and lymph nodes were substantially different compared with other sites, including bone, pleura and adrenal gland. The mPFS of patients with liver metastasis $(4.44 \pm 1.93$ months) was the least favorable, even shorter than that of patients with brain metastasis, as presented in Table I.

MICA prolongs the PFS time associated with chemotherapy. To detect the mRNAs of six NKG2DLs, the present study used paracancerous tissues as a control. Pre-treatment NKG2DL levels were determined in the tumor tissue samples of 115 patients with SCLC. The results indicated that MICA was closely associated with PFS time in patients treated with first-line chemotherapy (Pearson $\mathrm{r}=0.4496$; $\mathrm{P}<0.0001$; Fig. 1A). However, no significant associations were observed between PFS and the other five NKG2DLs. As presented in Fig. 1, the Pearson $r$ values of MICB, ULBP1, ULBP2, ULBP3 and ULBP4 were -0.0506, $0.0171,0.0120,0.0277$ and 0.1156 , respectively (all $\mathrm{P}>0.05$ ). Clinically, a small number of patients with drug-resistant SCLC relapse $<3$ months after the first treatment, which is defined as rapid chemoresistance (QCR) (33). In the present study, the 20 patients with the shortest PFS time were classified as having QCR, while the 20 patients with the longest PFS time were classified as having delayed chemotherapy resistance (DCR). The expression of the NKG2DLs were subsequently analyzed in these two groups of patients and the results demonstrated that MICA expression was significantly increased in the DCR group, in which MICA expression was 1.6 times higher compared with the QCR group (DCR, $1.5549 \pm 0.4506$ vs. QCR, $1.0000 \pm 0.3557$; $\mathrm{P}<0.0001$; Fig. 1G). However, the other NKG2DLs failed to demonstrate any significant differences between the two groups.

MICA significantly increases the sensitivity of tumor cells to platinum chemotherapy. MICA may improve the PFS of patients with SCLC, treated with chemotherapy; however, further investigations are required to verify whether this is caused by increased chemosensitivity. Thus, the present study constructed a NCI-H446 cell line which overexpressed MICA and verified the effect of MICA overexpression (Fig. S1). RT-qPCR analysis demonstrated that MICA expression in the overexpressed cell line was 3.103 times higher compared with the control group $(\mathrm{P}<0.001$; Fig. 2A).

Chemosensitivity of MICA-OE cells to carboplatin and cisplatin was subsequently assessed, with vector-only cells as the control. Overexpression of MICA significantly increased NCI-H446 chemosensitivity to cisplatin (Fig. 2B).
The $\mathrm{IC}_{50}$ value of cisplatin in NCI-H446 cells significantly decreased (vector cells, 9.279 $\pm 3.931 \mu \mathrm{mol} / 1$ vs. MICA-OE cells, 5.908 $\pm 2.775 \mu \mathrm{mol} / \mathrm{l}$; Fig. 2D). Furthermore, the chemosensitivity of MICA-OE cells to carboplatin also significantly increased. The $\mathrm{IC}_{50}$ value of carboplatin in the MICA-OE cells was $6.351 \pm 1.225 \mu \mathrm{mol} / 1$, while the $\mathrm{IC}_{50}$ value of carboplatin in the vector cells was $51.824 \pm 6.924 \mu \mathrm{mol} /$ (Fig. $2 \mathrm{C}$ and D). The results demonstrated that the $\mathrm{IC}_{50}$ value significantly decreased with overexpression of MICA, thus suggesting that MICA may increase the sensitivity of tumor cells to cisplatin and carboplatin.

ABCG2 serves a vital role in regulating the chemotherapy sensitivity induced by MICA. To understand the molecular mechanism by which MICA enhances chemosensitivity, the present study assessed the mRNA expression of 11 genes associated with chemosensitivity according to our previous research include: $\mathrm{ABCB} 1, \mathrm{ABCC} 1, \mathrm{ABCC} 2, \mathrm{ABCG} 2$, ABCC2, ABCC9, DPYD, GSTT1, GSTp1, GSTM1, ADH1A, PPARA (20). The primer sequences of the associated genes are listed in Table S1. The results demonstrated that overexpressing MICA downregulated the mRNA expression of ABCG2, ADH1 and GSTT1; however, no significant differences were observed in the mRNA levels of the other genes (ABCB1, ABCC1, ABCC2, ABCC9, DPYD, GSTT1, GSTp1 and GSTM1) (Fig. 3A). According to the GeneMANIA analysis, there is a complex interaction network between the NKG2DLs and chemotherapeutic sensitivity-associated genes (Fig. 3B). Western blot analysis was performed to determine the protein expression levels of ABCG2, ADH1 and GSTT1 in MICA-OE cells compared with the vector group. The results demonstrated that ABCG2 protein expression significantly decreased in the MICA-OE group compared with the control (Fig. 3C); however, there was no significant differences in ADH1 and GSTT1 protein expression between the two groups (Fig. 3D and E). ImageJ quantitative analysis of the western blots demonstrated that ABCG2 protein expression significantly decreased in the MICA-OE group compared with the control (Fig. 3F).

The results of western blotting were subsequently validated via chemosensitivity testing. ABCG2 blockers (nicardipine) was also added in order to clarify the role of ABCG2 in MICA chemotherapy sensitization (34). First, the effect on cell proliferation was examined to determine whether nicardipine improves the chemotherapeutic sensitivity of MICA-OE cells. A lower concentration of nicardipine $(10 \mu \mathrm{M})$ was selected as it has no significant effect on the proliferation of NCI-H446 cells (35). The MTT assay demonstrated a significantly lower $\mathrm{IC}_{50}$ value in the MICA-OE + nicardipine group compared with the vector group (Fig. 3G; vector group, $2.663 \pm 1.235 \mu \mathrm{M}$; vector + nicardipine group, $9.956 \pm 1.392 \mu \mathrm{M}$; MICA-OE + nicardipine group, 4.475 $\pm 1.178 \mu \mathrm{M}$; MICA-OE group, $9.279 \pm 1.931 \mu \mathrm{M})$

\section{Discussion}

In recent years, the molecular mechanism of the immune response against tumors has been recognized, along with the synergetic effect of immunotherapy in combination with chemotherapy (9). An in-depth understanding of the role of 
A
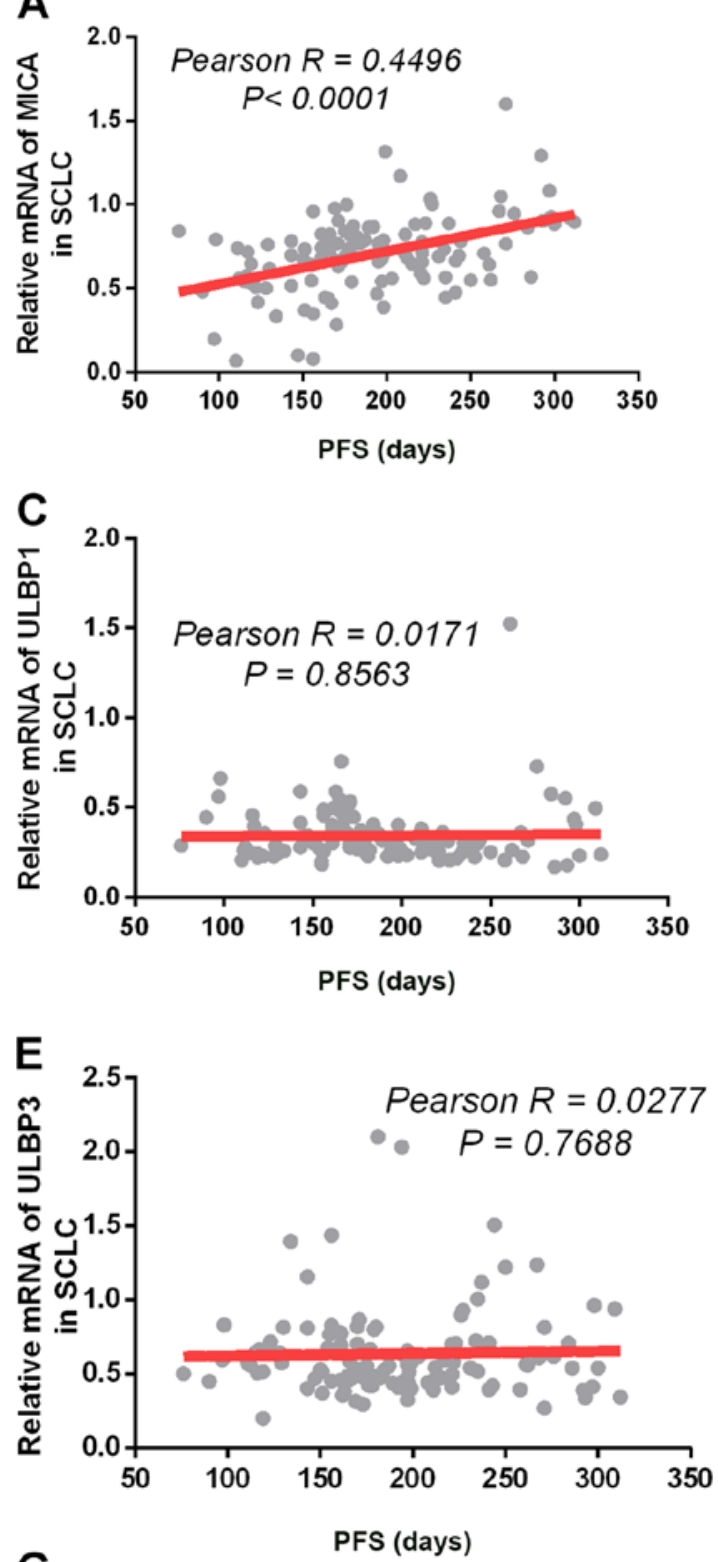

G

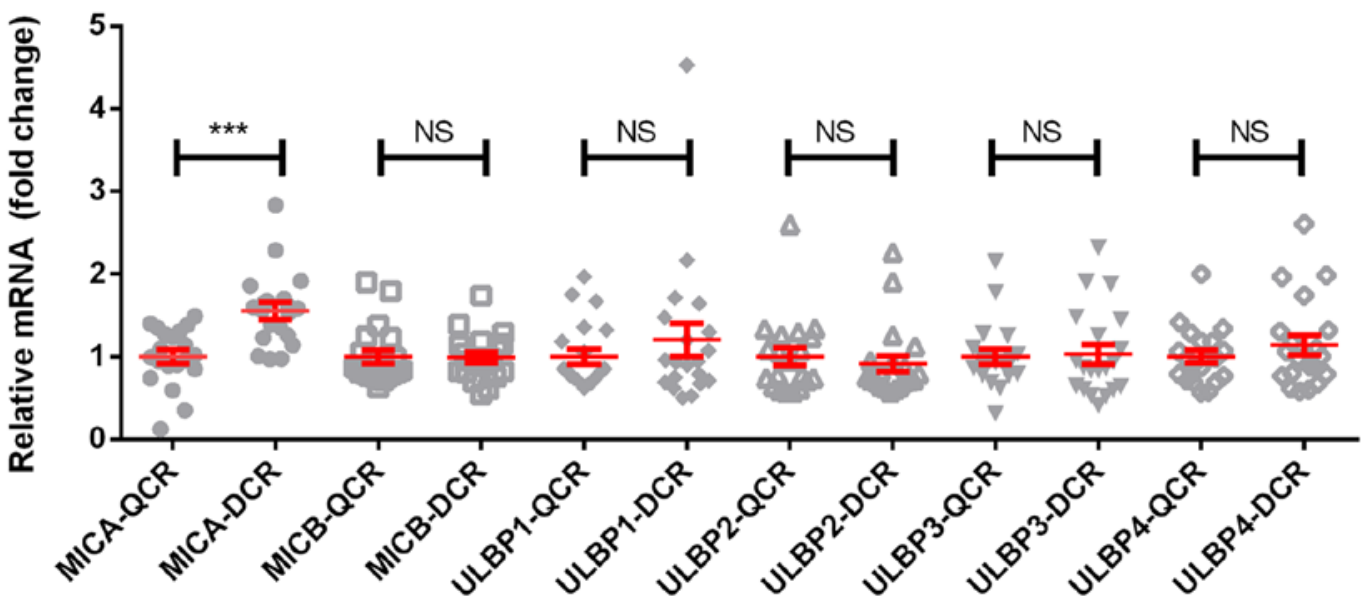

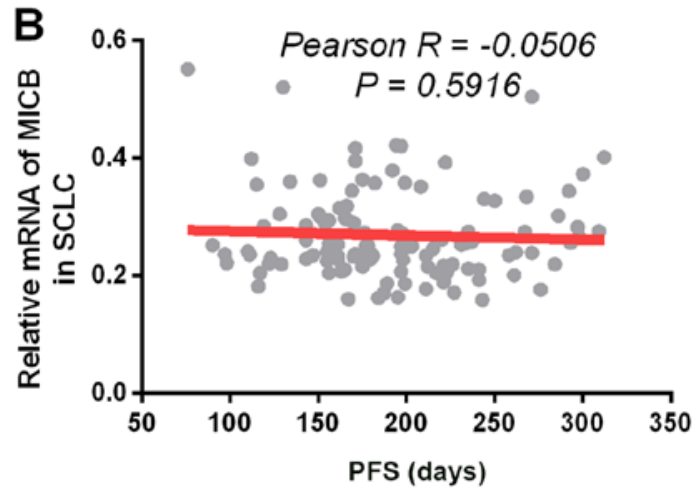
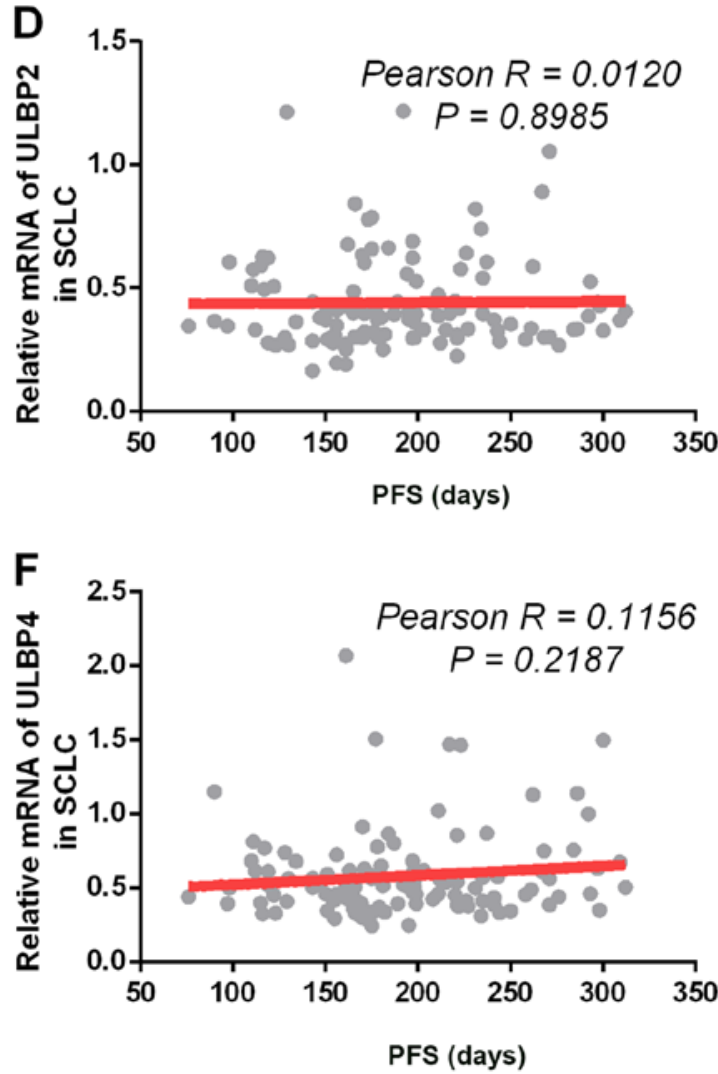

Figure 1. Association between NKG2DLs and PFS in SCLC treated with first-line chemotherapy. (A) MICA mRNA expression was significantly associated with PFS in SCLC treated with first-line chemotherapy. (B) No significant association was observed between PFS and MICB. (C) No significant association was observed between PFS and ULBP1. (D) No significant association was observed between PFS and ULBP2. (E) No significant association was observed between PFS and ULBP3. (F) No significant association was observed between PFS and ULBP4. (G) MICA expression significantly increased in the DCR group compared with the QCR group. ${ }^{* * *} \mathrm{P}<0.001$ MICA-QCR vs. MICA-DCR. NKG2DLs, natural killer cell-activated receptor natural-killer group-2 member D ligands; PFS, progression-free survival; SCLC, small cell lung cancer; MICA, major histocompatibility complex class I polypeptide-related sequence A; QCR, quick chemotherapy resistance; DCR, delayed chemotherapy resistance; ULBP, UL16-binding protein; NS, nonsignificant. 
A

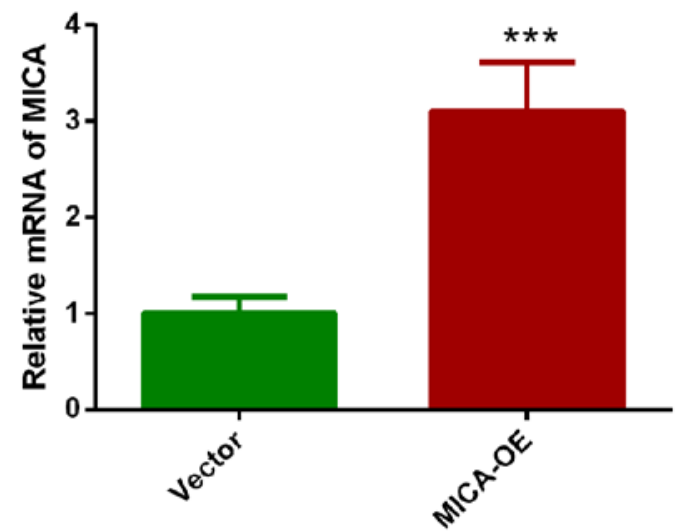

C

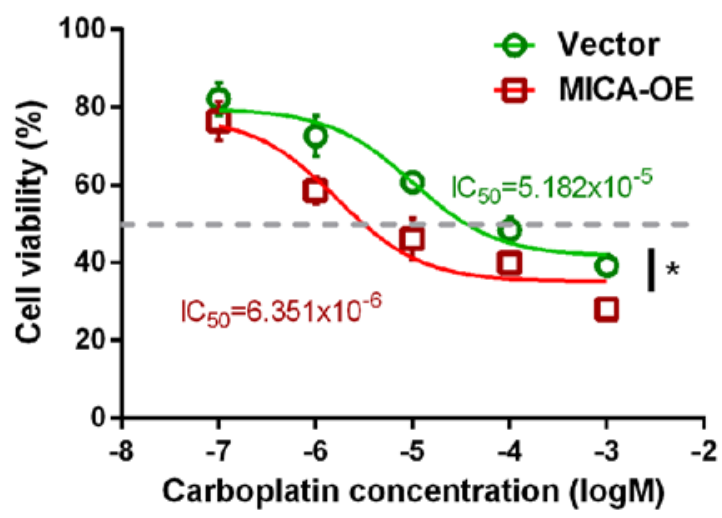

B

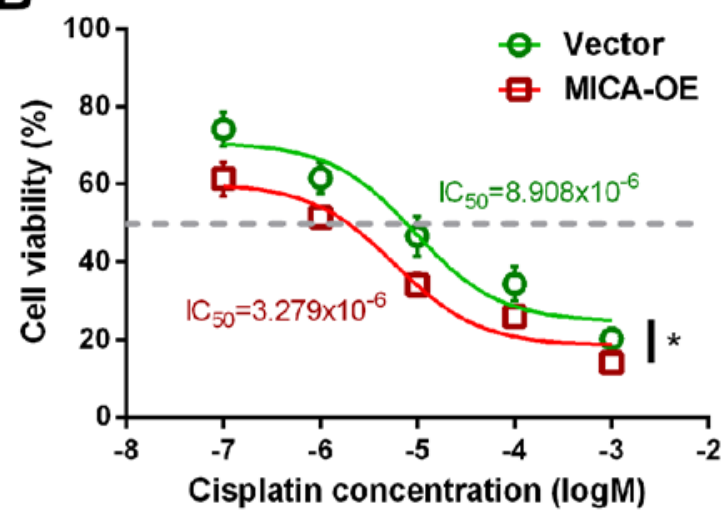

D

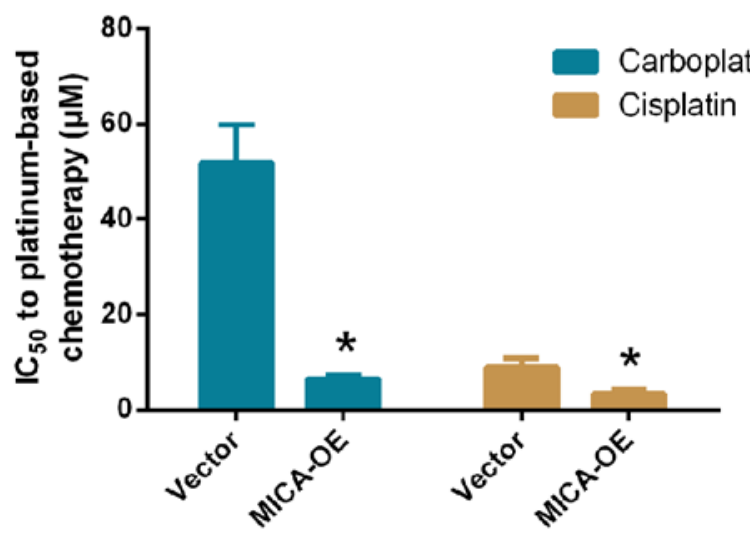

Figure 2. Overexpression of MICA increases the chemosensitivity of tumor cells. (A) Following lentiviral transfection in NCI-H446 cells, MICA expression increased by 3.103-fold in the MICA-OE group compared with the Vector group. (B) The $\mathrm{IC}_{50}$ of carboplatin in the MICA-OE group significantly decreased compared with that in the Vector. (C) The $\mathrm{IC}_{50}$ of cisplatin in the MICA-OE group was significantly lower than that in the control group. (D) Cell viability was compared using ANOVA at $10 \mu \mathrm{mol} / 1$ carboplatin concentration. ${ }^{*} \mathrm{P}<0.05 \mathrm{MICA}-\mathrm{OE}$ vs. vector, and ${ }^{* * *} \mathrm{P}<0.001 \mathrm{mRNA}$ of $\mathrm{MICA}-\mathrm{OE}$ vs. vector. MICA-OE, major histocompatibility complex class I polypeptide-related sequence A overexpression; $\mathrm{IC}_{50}$, the half maximal inhibitory concentration; ABCG2, ATP binding cassette subfamily G member 2; ADH1, alcohol dehydrogenase 1A (class I), alpha polypeptide; GSTT1, glutathione S-transferase theta 1.

NKG2D in tumor chemotherapy may contribute to the establishment of a novel clinical therapy for SCLC. NKG2DLs serve an essential role in the process of immune recognition and cell killing mediated by NK cells, and they are also crucial to the antitumor response (36).

Previous research on immune drugs has focused on the effect on the tumor itself, without considering its interaction with chemotherapeutic agents. The expression of NKG2DLs are lower in tumor tissues compared with healthy tissues (10), which was confirmed in the present study. Furthermore, this decreased expression is also considered to be a molecular mechanism underlying tumor immune escape $(37,38)$. Zhang et al (39) demonstrated that the expression of NKG2DLs by tumor cells may result in the efficient development of antitumor immune responses in vitro and in vivo (39). The present study assessed MICA expression in patients with SCLC by determining the association between MICA expression levels and PFS time and confirmed that it may enhance the sensitivity of SCLC to platinum chemotherapy. MICA serves a crucial role in the tumor chemotherapy response, indicating that high MICA has a synergistic effect on chemotherapy. Thus, MICA may represent a predictive factor for the efficacy of chemotherapy and serve as a prognostic marker for patients receiving chemotherapy.

The results of the present study demonstrated that overexpressing MICA in vitro increased the sensitivity of cells to cisplatin and carboplatin. This phenomenon may be due to three factors: i) High MICA expression enhances the tumor killing activity of NK and T cells (40); ii) increased numbers of $\mathrm{NK}$ and $\mathrm{T}$ cells demonstrate a more specific antitumor effect, particularly during chemotherapy and tumor necrosis antigen exposure (41); and iii) high NKG2D expression decreases the efflux pump activity of the tumor cell membrane, while increasing the intracellular concentration of drugs, which may enhance the chemosensitivity of tumor cells.

To determine the molecular mechanism, the expression of 11 genes in tumor specimens from patients with SCLC were assessed. The expression changes associated with drug sensitivity in tumor tissues with high MICA expression were predominantly concentrated in the ATP binding cassette protein transport pathway genes, while the expression changes associated with drug metabolism were primarily concentrated in the glutathione S-transferase pathway genes. RT-qPCR and western blot analyses were performed to verify mRNA and protein expression in MICA-overexpressing 


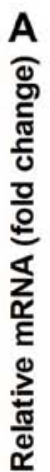
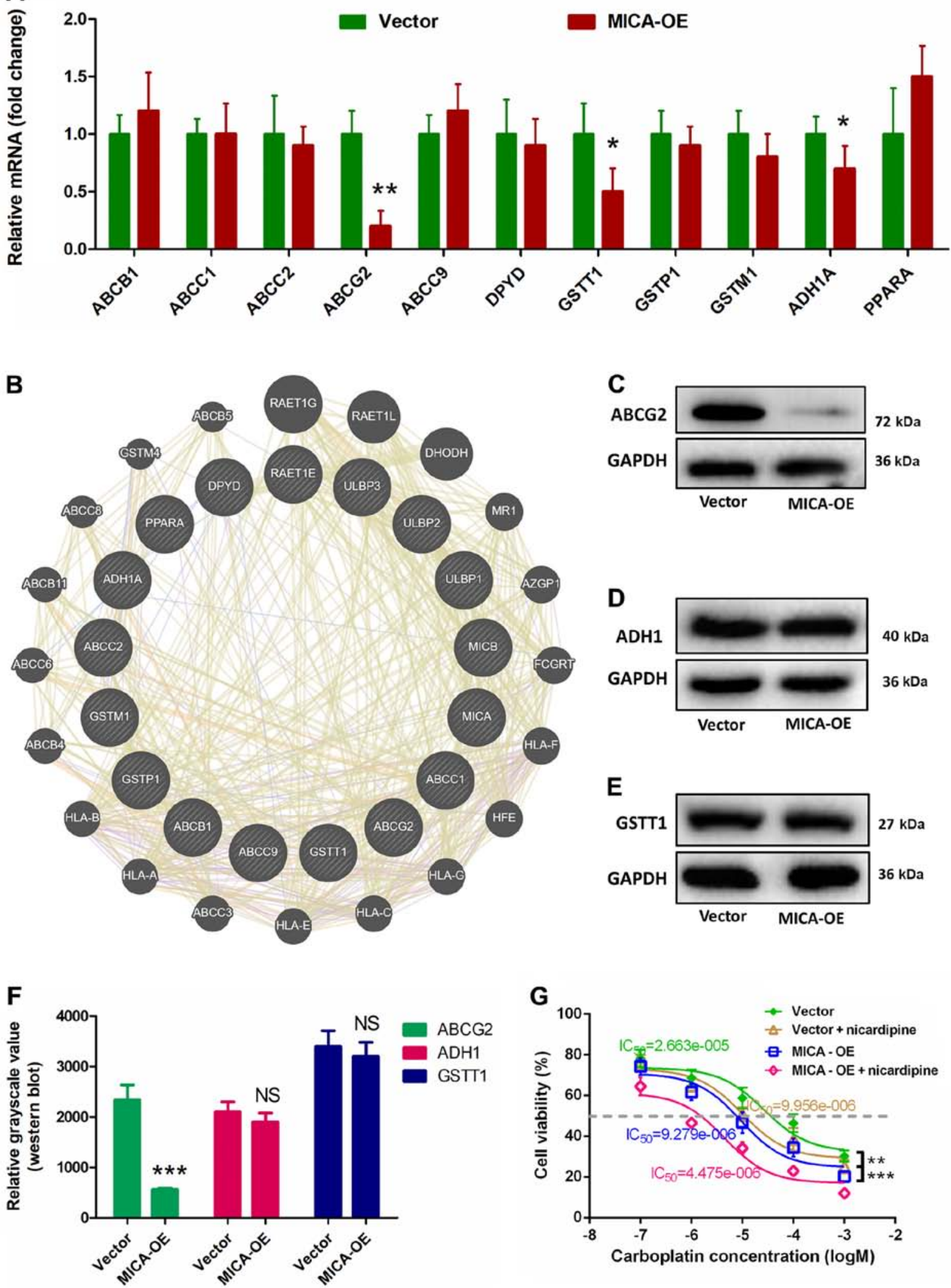

Figure 3. ABCG2 serves a crucial role in MICA-induced chemosensitivity. (A) mRNA expression levels of ABCG2, ADH1A and GSTT1 were significantly downregulated compared with the Vector control group. (B) According to the GeneMANIA analysis, there is a complex interaction network between NKG2DLs and chemotherapeutic sensitivity-associated genes. (C) ABCG2 protein expression significantly decreased in the MICA overexpression group, while (D) ADH1 and (E) GSTT1 protein expression levels did not. (F) ImageJ western blot quantification suggested that ABCG2 expression was downregulated considerably compared with the Vector group. (G) MICA-induced chemosensitivity significantly increased following addition of nicardipine. Cell viability was compared using ANOVA, at $10 \mu \mathrm{mol} / 1$ of carboplatin. ${ }^{*} \mathrm{P}<0.05$, mRNA of GSTT1 and ADH1A in MICA-OE vs. vector. ${ }^{* *} \mathrm{P}<0.01$, mRNA of ABCG2 in MICA-OE vs. vector (A) or cell viability of MICA-OE vs. vector in response to $10^{-5} \mathrm{M}$ carboplatin $(\mathrm{G}){ }^{* * *} \mathrm{P}<0.001$, grayscale value of $\mathrm{ABCG} 2$ in MICA-OE vs. vector (F), or cell viability of MICA-OE + nicardipine vs. vector in resopnse to $10^{-5} \mathrm{M}$ carboplatin (G). ABCG2, ATP binding cassette subfamily G member 2; MICA, major histocompatibility complex class I polypeptide-related sequence A; ADH1A, alcohol dehydrogenase 1A (class I), $\alpha$ polypeptide; GSTT1, glutathione S-transferase $\theta 1$. 
tumor cells, respectively. The results demonstrated that ABCG2 expression decreased in tumor cells overexpressing MICA. This result supports the hypothesis that high NKG2D expression decreases the activity of the efflux pump, while increasing the intracellular concentration of drugs. However, the present study has some limitations. Firstly, a major limitation of the present study is that only one SCLC cell line was used to verify the results. Secondly, the experimental results were not verified using animal models and clinically in patients. In subsequent studies, the growth rate of MICA-expressing multi-tumor cells in mice and the response to chemotherapy need to be studied. Subsequently, the key genes in the upstream and downstream pathways of MICA will also be sequenced in whole genes.

Previous studies have confirmed that downregulation of ABCG2 serves a vital role in resistance to chemotherapy $(35,42,43)$. Membrane transport proteins belong to the ATP-binding cassette transport protein superfamily $(42,43)$. Members of this superfamily utilize energy from ATP hydrolysis to transport a number of substrates, including peptides, lipids and anticancer drugs, across the membrane. The human genome contains 48 ATP binding cassette subfamily genes, which can be further divided into seven different subfamilies, named A-G, according to sequence homology and domain similarity (42). These proteins mediate the development of multidrug resistance in several types of cancer cells, including breast and colon cancer (44-47). ABCG2 transporter protein is a key ABC protein, which is ubiquitously expressed in lung and liver, and contributes to the disposition of a variety of endogenous substances, including platinum-based regimens $(48,49)$. Previous studies have demonstrated an association between ABCG2 expression and prognosis and survival in patients with SCLC cancer $(45,46,50)$. Peptides generated by the ubiquitin-proteasome pathway, including MICA, are presented by MHC class I molecules (51). The predominant function of the MIC protein family is to display peptides to cytotoxic $\mathrm{CD}^{+} \mathrm{T}$ cells in support of their crucial activity of immune surveillance (36). Thus, the present study hypothesized that MICA decreases ABCG2 expression on the cell membrane via antigen presentation. ABCG2 functions as a xenobiotic transporter and its involvement in multi-drug resistance has been well-established and previously validated $(35,52)$. For exclude other pathway caused by MICA-mediated drug resistance, nicardipine was selected to be the next phase of the experiment as strong inhibitors of $\mathrm{ABCG} 2$. As expected, the $\mathrm{IC}_{50}$ of MICA-OE was significant down-regulation by nicardipine in SCLC cells to carboplatin. The results of the present study confirmed that MICA is closely associated with the expression of membrane transport proteins in tumors and that chemosensitivity can be achieved by downregulating ABCG2. However, the molecular mechanisms by which overexpression of MICA decreases ABCG2 expression remain unclear, thus further investigation is required.

Currently, the focus is on programmed cell death-1 and programmed death-ligand 1 , whereby several clinical trials have been performed using immunotherapy combined with chemotherapy (6). Most of these clinical studies have achieved positive results; however, they have predomi- nantly prompted researchers to better investigate immune system-enhancing drugs and assess the mechanism of synergy with chemotherapy.

To the best of our knowledge the present study was the first to identify an association between immunotherapy and chemotherapy in patients with SCLC. Taken together, the results of the present study suggest that MICA may be used as a marker to predict the response to chemotherapy and the prognosis of patients with SCLC and it may improve sensitivity to cisplatin chemotherapy via downregulation of ABCG2 expression.

\section{Acknowledgements}

The authors of the present study would like to thank Ms. Suxia Luo from the Affiliated Cancer Hospital of Zhengzhou University, for valuable guidance in the initial stages of writing the manuscript. The authors would also like to acknowledge and Ms. Shujun Yang from the Affiliated Cancer Hospital of Zhengzhou University for his help in the patient's investigation.

\section{Funding}

The present study was funded by the Science and Technology Program of Henan Province (grant no. 162102310327), the Natural Science Foundation of Henan Province (grant nos. 182300410297, 182300410297 and 162300410300), the Medical Science and Technology Program of Henan Province (grant no. 201702249) and the 51282 Project Leading Talent of Henan Provincial Health Science and Technology Innovation Talents (grant no. [2016]32).

\section{Availability of data and materials}

The datasets used and/or analyzed during the current study are available from the corresponding author on reasonable request.

\section{Authors' contributions}

YW wrote the manuscript. YW and HT performed data analysis. RS, SX, QW and YW conceived the study. RS and SX designed the study. RW and QW collected the data. All authors read and approved the final manuscript.

\section{Ethics approval and consent to participate}

The present study was approved by the Ethics Committee of Zhengzhou University (approval no. 2014079) and performed in accordance with the The Declaration of Helsinki. Written informed consent was provided by all patients prior to the study start.

\section{Patient consent for publication}

Not applicable.

\section{Competing interests}

The authors declare that they have no competing interests. 


\section{References}

1. Cao M and Chen W: Epidemiology of lung cancer in China Thorac Cancer 10: 3-7, 2019.

2. Kalemkerian GP: Small Cell Lung Cancer. Semin Respir Crit Care Med 37: 783-796, 2016.

3. PeltonK,CoticchiaCM,CuratoloAS,SchaffnerCP,ZurakowskiD, Solomon KR and Moses MA: Hypercholesterolemia induces angiogenesis and accelerates growth of breast tumors in vivo. Am J Pathol 184: 2099-2110, 2014.

4. Ott PA, Elez E, Hiret S, Kim DW, Morosky A, Saraf S, Piperdi B and Mehnert JM: Pembrolizumab in patients with extensive-stage small-cell lung cancer: Results from the phase Ib KEYNOTE-028 Study. J Clin Oncol 35: 3823-3829, 2017.

5. Fan Y and Mao W: Immune checkpoint inhibitors in lung cancer: Current status and future directions. Chin Clin Oncol 6: 17, 2017.

6. Tanvetyanon T, Gray JE and Antonia SJ: PD-1 checkpoint blockade alone or combined PD-1 and CTLA-4 blockade as immunotherapy for lung cancer? Expert Opin Biol Ther 17: 305-312, 2017.

7. Ready N, Farago AF, de Braud F, Atmaca A, Hellmann MD, Schneider JG, Spigel DR, Moreno V, Chau I, Hann CL, et al: Third-line nivolumab monotherapy in recurrent SCLC: CheckMate 032. J Thorac Oncol 14: 237-244, 2019.

8. Souza-Fonseca-Guimaraes F, Cursons J and Huntington ND: The emergence of natural killer cells as a major target in cancer immunotherapy. Trends Immunol 40: 142-158, 2019.

9. Cai X, Caballero-Benitez A, Gewe MM, Jenkins IC Drescher CW, Strong RK, Spies T and Groh V: Control of tumor initiation by NKG2D naturally expressed on ovarian cancer cells. Neoplasia 19: 471-482, 2017.

10. Bauer S, Groh V, Wu J, Steinle A, Phillips JH, Lanier LL and Spies T: Activation of NK cells and T cells by NKG2D, a receptor for stress-inducible MICA. Science 285: 727-729, 1999.

11. Diefenbach A, Jamieson AM, Liu SD, Shastri N and Raulet DH: Ligands for the murine NKG2D receptor: Expression by tumor cells and activation of NK cells and macrophages. Nat Immunol 1: 119-126, 2000.

12. Cosman D, Mullberg J, Sutherland CL, Chin W, Armitage R, Fanslow W, Kubin M and Chalupny NJ: ULBPs, novel MHC class I-related molecules, bind to CMV glycoprotein UL16 and stimulate NK cytotoxicity through the NKG2D receptor. Immunity 14: 123-133, 2001.

13. Eagle RA, Traherne JA, Hair JR, Jafferji I and Trowsdale J: ULBP6/RAET1L is an additional human NKG2D ligand. Eur $\mathbf{J}$ Immunol 39: 3207-3216, 2009.

14. Liu H, Wang S, Xin J, Wang J, Yao C and Zhang Z: Role of NKG2D and its ligands in cancer immunotherapy. Am J Cancer Res 9: 2064-2078, 2019.

15. Lopez-Soto A, Huergo-Zapico L, Acebes-Huerta A, Villa-Alvarez $M$ and Gonzalez S: NKG2D signaling in cancer immunosurveillance. Int J Cancer 136: 1741-1750, 2015.

16. Lanier LL: NKG2D Receptor and Its Ligands in Host Defense. Cancer Immunol Res 3: 575-582, 2015.

17. Inagaki A, Ishida T, Yano H, Ishii T, Kusumoto S, Ito A, Ri M, Mori F, Ding J, Komatsu H, et al: Expression of the ULBP ligands for NKG2D by B-NHL cells plays an important role in determining their susceptibility to rituximab-induced ADCC. Int J Cancer 125: 212-221, 2009.

18. Travis WD, Brambilla E, Burke AP, Marx A and Nicholson AG Introduction to The 2015 World Health Organization classification of tumors of the lung, pleura, thymus, and heart. J Thorac Oncol 10: 1240-1242, 2015

19. Eisenhauer EA, Therasse P, Bogaerts J, Schwartz LH, Sargent D, Ford R, Dancey J, Arbuck S, Gwyther S, Mooney M, et al: New response evaluation criteria in solid tumours: Revised RECIST guideline (version 1.1). Eur J Cancer 45: 228-247, 2009.

20. Fan Y, Gan Y, Shen Y, Cai X, Song Y, Zhao F, Yao M, Gu J and Tu H: Leptin signaling enhances cell invasion and promotes the metastasis of human pancreatic cancer via increasing MMP-13 production. Oncotarget 6: 16120-16134, 2015.

21. Wu Y, Gan Y, Yuan H, Wang Q, Fan Y, Li G, Zhang J, Yao M, $\mathrm{Gu} \mathrm{J}$ and $\mathrm{Tu} \mathrm{H}$ : Enriched environment housing enhances the sensitivity of mouse pancreatic cancer to chemotherapeutic agents. Biochem Biophys Res Commun 473: 593-599, 2016.

22. Livak KJ and Schmittgen TD: Analysis of relative gene expression data using real-time quantitative PCR and the 2(-Delta Delta C(T)) method. Methods 25: 402-408, 2001.
23. Barrett T, Troup DB, Wilhite SE, Ledoux P, Rudnev D, Evangelista C, Kim IF, Soboleva A, Tomashevsky M, Marshall KA, et al: NCBI GEO: Archive for high-throughput functional genomic data. Nucleic Acids Res 37: 885-890, 2009.

24. Breitkreutz BJ, Stark C, Reguly T, Boucher L, Breitkreutz A, Livstone M, Oughtred R, Lackner DH, Bähler J, Wood V, et al: The BioGRID Interaction Database: 2008 update. Nucleic Acids Res 36: D637-D640, 2008.

25. Brown KR and Jurisica I: Online predicted human interaction database. Bioinformatics 21: 2076-2082, 2005.

26. Keshava Prasad TS, Goel R, Kandasamy K, Keerthikumar S, Kumar S, Mathivanan S, Telikicherla D, Raju R, Shafreen B, Venugopal A, et al: Human protein reference database-2009 update. Nucleic Acids Res 37: 767-772, 2009.

27. Romero P, Wagg J, Green ML, Kaiser D, Krummenacker M and Karp PD: Computational prediction of human metabolic pathways from the complete human genome. Genome Biol 6: R2, 2005.

28. Aranda B, Achuthan P, Alam-Faruque Y, Armean I, Bridge A, Derow C, Feuermann M, Ghanbarian AT, Kerrien S, Khadake J, et al: The IntAct molecular interaction database in 2010. Nucleic Acids Res 38: D525-D531, 2010.

29. Ceol A, Chatr Arayamontri A, Licata L, Peluso D, Briganti L, Perfetto L, Castagnoli L and Cesareni G: MINT, the molecular interaction database: 2009 update. Nucleic Acids Res 38: D532-D539, 2010.

30. Schaefer CF, Anthony K, Krupa S, Buchoff J, Day M, Hannay T and Buetow KH: PID: The pathway interaction database. Nucleic Acids Res 37: D674-D679, 2009.

31. Vastrik I, D'Eustachio P, Schmidt E, Gopinath G, Croft D, de Bono B, Gillespie M, Jassal B, Lewis S, Matthews L, et al: Reactome: A knowledge base of biologic pathways and processes. Genome Biol 8: R39, 2007.

32. Warde-Farley D, Donaldson SL, Comes O, Zuberi K, Badrawi R, Chao P, Franz M, Grouios C, Kazi F, Lopes CT, et al: The GeneMANIA prediction server: Biological network integration for gene prioritization and predicting gene function. Nucleic Acids Res 38 (Web Server issue): W214-W220, 2010.

33. Wu Y, Si R, Tang H, He Z, Zhu H, Wang L, Fan Y, Xia S, He Z and Wang Q: Cholesterol reduces the sensitivity to platinum-based chemotherapy via upregulating ABCG2 in lung adenocarcinoma. Biochem Biophys Res Commun 457: 614-620, 2015.

34. Shukla S, Robey RW, Bates SE and Ambudkar SV: The calcium channel blockers, 1,4-dihydropyridines, are substrates of the multidrug resistance-linked ABC drug transporter, ABCG2. Biochemistry 45: 8940-8951, 2006.

35. Wu Y, Si R, Yang S, Xia S, He Z, Wang L, He Z, Wang Q and Tang H: Depression induces poor prognosis associates with the down-regulation brain derived neurotrophic factor of serum in advanced small cell lung cancer. Oncotarget 7: 85975-85986, 2016.

36. Hu J, Zhu S, Xia X, Zhang L, Kleinerman ES and Li S: CD8+T cell-specific induction of NKG2D receptor by doxorubicin plus interleukin-12 and its contribution to CD8+T cell accumulation in tumors. Mol Cancer 13: 34, 2014.

37. Raffaghello L, Prigione I, Airoldi I, Camoriano M, Levreri I, Gambini C, Pende D, Steinle A, Ferrone S and Pistoia V: Downregulation and/or release of NKG2D ligands as immune evasion strategy of human neuroblastoma. Neoplasia 6: 558-568, 2004.

38. Dhar P and Wu JD: NKG2D and its ligands in cancer. Curr Opin Immunol 51: 55-61, 2018.

39. Zhang J, Basher F and Wu JD: NKG2D ligands in tumor immunity: Two sides of a coin. Front Immunol 6: 97, 2015.

40. Deng W, Gowen BG, Zhang L, Wang L, Lau S, Iannello A, Xu J, Rovis TL, Xiong N and Raulet DH: Antitumor immunity. A shed NKG2D ligand that promotes natural killer cell activation and tumor rejection. Science 348: 136-139, 2015.

41. Zen K, Karsan A, Stempien-Otero A, Yee E, Tupper J, Li X, Eunson T, Kay MA, Wilson CB, Winn RK and Harlan JM: NF-kappaB activation is required for human endothelial survival during exposure to tumor necrosis factor-alpha but not to interleukin-1beta or lipopolysaccharide. J Biol Chem 274: 28808-28815, 1999.

42. Theodoulou FL and Kerr ID: ABC transporter research: Going strong 40 years on. Biochem Soc Trans 43: 1033-1040, 2015.

43. Boswell-Casteel RC, Fukuda Y and Schuetz JD: ABCB6, an $\mathrm{ABC}$ transporter impacting drug response and disease. AAPS J 20: 8, 2017. 
44. Gillet JP and Gottesman MM: Advances in the molecular detection of $\mathrm{ABC}$ transporters involved in multidrug resistance in cancer. Curr Pharm Biotechnol 12: 686-692, 2011.

45. Huang Y, Penchala S, Pham AN and Wang J: Genetic variations and gene expression of transporters in drug disposition and response. Expert Opin Drug Metab Toxicol 4: 237-254, 2008.

46. Zinzi L, Capparelli E, Cantore M, Contino M, Leopoldo M and Colabufo NA: Small and innovative molecules as new strategy to revert MDR. Front Oncol 4: 2, 2014.

47. Colabufo NA, Berardi F, Contino M, Niso M and Perrone R: ABC pumps and their role in active drug transport. Curr Top Med Chem 9: 119-129, 2009.

48. Xie N, Mou L, Yuan J, Liu W, Deng T, Li Z, Jing Y and Hu Z Modulating drug resistance by targeting BCRP/ABCG2 using retrovirus-mediated RNA interference. PLoS One 9: e103463, 2014.

49. de Boussac H, Orban TI, Varady G, Tihanyi B, Bacquet C, Brózik A, Váradi A, Sarkadi B and Arányi T: Stimulus-induced expression of the ABCG2 multidrug transporter in HepG2 hepatocarcinoma model cells involves the ERK1/2 cascade and alternative promoters. Biochem Biophys Res Commun 426: 172-176, 2012.
50. Benderra Z, Faussat AM, Sayada L, Perrot JY, Chaoui D, Marie JP and Legrand O: Breast cancer resistance protein and P-glycoprotein in 149 adult acute myeloid leukemias. Clin Cancer Res 10: 7896-7902, 2004.

51. Yewdell JW: Not such a dismal science: The economics of protein synthesis, folding, degradation and antigen processing. Trends Cell Biol 11: 294-297, 2001.

52. Patel A, Li TW, Anreddy N, Wang DS, Sodani K, Gadhia S Kathawala R, Yang DH, Cheng C and Chen ZS: Suppression of ABCG2 mediated MDR in vitro and in vivo by a novel inhibitor of ABCG2 drug transport. Pharmacol Res 121: 184-193, 2017.

This work is licensed under a Creative Commons Attribution-NonCommercial-NoDerivatives 4.0 International (CC BY-NC-ND 4.0) License. 